\title{
Bright White-Light Emitting Manganese and Copper Co-Doped ZnSe Quantum Dots**
}

\author{
Subhendu K. Panda, Stephen G. Hickey,* Hilmi Volkan Demir, and Alexander Eychmüller
}

White-light emission (WLE) from semiconductor nanostructures is presently a research area of intense interest, especially where the primary objective is to replace conventional light sources in order to minimize energy costs and therefore global energy consumption for lighting. ${ }^{[1-8]}$ Presently the general methods to achieve white-light emission are either by coating a yellow phosphor or by combining green and red phosphors on a background consisting of a blue-light emitting diode (LED) or by employing nanocrystals (NCs) of the three primary colors (red, green, blue) using multilayer structures in LEDs. However, when one simply mixes these nanocrystal quantum dots (QDs) of different colors together to generate white light, the efficiencies are often observed to decrease due to the re-absorption of light and subsequent undesired energy transfer (ET). This may lead to undesirable changes in the chromaticity coordinates and photometric performance due to the different relative temporal stabilities of the components. Hence the use of a single-emitting component offers many advantages over multiple component systems for whitelight emitting sources such as LEDs, amongst which are: greater reproducibility, low cost preparation, ease of modification, and simpler fabrication processes. Therefore, it is of great importance for many applications to find high-quality single source white-light emitters through low-cost chemical synthesis approaches that will allow the production of white light while meeting the needs of industry, such as satisfactory Commission International d'Eclairage (CIE) coordinates.

One route that offers the possibility by which such materials may be accessed is that of the colloidal synthesis of doped semiconductor nanocrystals, which has already proven itself to be an interesting field for future nanotechnologies as it can presently provide highly efficient emission sources for various applications. ${ }^{[9-12]}$ Although

[*] Dr. S. K. Panda, Dr. S. G. Hickey, Prof. A. Eychmüller

Physical Chemistry/Electrochemistry, TU Dresden

Bergstrasse 66b, 01062 Dresden (Germany)

Fax: (+49) 351-463-37164

E-mail: s.hickey@chemie.tu-dresden.de

Prof. H. V. Demir

Department of Electrical and Electronics Engineering, Department of Physics, UNAM—National Nanotechnology Research Center and Institute of Materials Science and Nanotechnology, Bilkent University, Ankara 06800 (Turkey)

[**: S.K.P. is grateful for a research fellowship provided by the Alexander von Humboldt Foundation. S.G.H. and H.V.D. acknowledge financial support through the FP7 Network of Excellence "Nanophotonics4Energy" and BMBF/TUBITAK joint project No. TUR09/ 001 and TUBITAK EEEAC No. $109 E 002$.

(9) Supporting information for this article is available on the WWW under http://dx.doi.org/10.1002/anie.201100464. various attempts have been made towards the growth of doped QDs in solution, it still remains a challenge to dope all of the nanocrystals present in the reaction mixture simultaneously with the different dopants, as the host matrix tends to expel the dopant ions from the internal crystal lattice to the surface, in a sort of "self-purification" process. ${ }^{[13]}$ Therefore, even in the most favorable cases of the dopant ions having the same valence state and similar ionic radius as those of the corresponding host, successful doping remains difficult to achieve by the simple addition of a small amount of dopant precursors during the synthesis of the host NCs. To overcome this, a number of doping strategies, such as nucleation-doping and growth-doping, where the doping is decoupled from the nucleation and/or growth, provide ample possibilities to selectively introduce dopants at desired positions within the host materials to generate different emission centers inside of a single quantum dot. ${ }^{[14]}$ Usually, in a $\mathrm{Mn}^{2+}$-doped $\mathrm{ZnSe}$ system there is a dominant yellow/orange emission present at $585 \mathrm{~nm}$ which results from the ${ }^{4} \mathrm{~T}_{1}-{ }^{6} \mathrm{~A}_{1}$ transition of the $\mathrm{Mn}^{2+}$ impurity excited by energy transfer from the host lattice. ${ }^{[15,16]}$ Therefore, if one can supply a source of blue and/or green emission within such a system, then white-light emission is likely to result. In fact, $\mathrm{Mn}^{2+}$-doped $\mathrm{CdS}^{[17]}$ and $\mathrm{ZnS}^{[18,19]} \mathrm{NCs}$ with white-light emission have been successfully prepared by the combination of the orange emission of the $\mathrm{Mn}^{2+}$ impurity and the blue and/or green emission of the surface defect states of the NCs. There are also a number of reports which describe the synthesis of semiconductor NCs with white-light emission such as "magic-sized" CdSe NCs, ${ }^{[1]}$ trap-rich CdS-QDs and onion-like CdSe/ZnS/CdSe/ZnS-QDs, ${ }^{[2]}$ alloyed $\mathrm{Zn}_{x} \mathrm{Cd}_{1-x} \mathrm{Se}$ quantum dots, ${ }^{[3]} \mathrm{ZnS}: \mathrm{Pb},{ }^{[4]} \mathrm{ZnS}$ incorporated into porous Silicon, ${ }^{[5]}$ and $\mathrm{ZnSe}^{[6]}$ However, all the above-mentioned systems rely on the manipulation of surface-state emission from the NCs, which is notoriously difficult to control and/or reproduce and, in addition, the temporal stability of these states varies with the environmental conditions in a manner which is presently still not fully understood. Also the intrinsic toxicity of cadmium and lead sheds a doubt on the future applicability of these NCs, particularly in view of recent environmental regulations.

Herein we report a method which overcomes these difficulties through the successful synthesis of doubly doped QDs using a versatile hot-injection colloidal synthesis to produce $\mathrm{Mn}$ and $\mathrm{Cu}$ co-doped $\mathrm{ZnSe}$ QDs (Cu:Mn-ZnSe), where white-light emission can be readily realized and its characteristics tuned. The two dopants have been introduced into the host material in a two-step process such that the dopants retain their individual emission properties which cover most of the visible spectral range. Also we demonstrate versatility of the tuning of the white-light generation with 
respect to its adjustable tristimulus coordinates, correlated color temperature and color rending index. The synthesized doped QDs also fulfill the potential of semiconductor QDs without the toxicity limitations encountered by II-VI QDs, therefore this material may offer the opportunity for futuregeneration WLE materials. To achieve co-doping of the $\mathrm{ZnSe}$ host with $\mathrm{Mn}$ and $\mathrm{Cu}$, the dopants were introduced at different times of the host growth. Details of a typical synthesis are provided in the Supporting Information.

Figure 1 shows the photoluminescence (PL) and optical absorbance spectra of the samples obtained at different stages of the synthesis. In the first step core-doped Mn-ZnSe QDs were synthesized by a nucleation-doping strategy, and in the second step subsequent incorporation of the $\mathrm{Cu}$ ions into the doped QDs was achieved. The initial MnSe nanoclusters were synthesized by the reaction of manganese stearate, $\mathrm{Mn}(\mathrm{St})_{2}$, and seleniumtributylphosphine in an amine-rich solution, the reaction mixture being kept at $280^{\circ} \mathrm{C}$ for 30 to $45 \mathrm{~min}$ due to the low reactivity of the manganese precursor. The use of larger amounts of amine was also found to be advantageous for the growth of $\mathrm{ZnSe}$ as it controls the reactivity of the zinc fatty acid/salt fatty acid mixture in the reaction mixture. Upon addition of the $\mathrm{Zn}$ precursor to the synthesized $\mathrm{MnSe}$ nanocrystals, the appearance of a yellow emission was observed under UV illumination.

From Figure $1 \mathrm{a}$ it can be seen that three distinct peaks may be observed for the $\mathrm{Mn}-\mathrm{ZnSe}$ doped QDs. The highly intense yellow emission at $585 \mathrm{~nm}$ results from the ${ }^{4} \mathrm{~T}_{1}-{ }^{6} \mathrm{~A}_{1}$ transition of the $\mathrm{Mn}^{2+}$ impurity excited by energy transfer from the host $\mathrm{ZnSe} .^{[15,16]}$ The peak at $410 \mathrm{~nm}$ and the broad peak at $470 \mathrm{~nm}$ are due to band-edge emission and surface defects, respectively, which emanate from the host $\mathrm{ZnSe}$. It was possible to observe these $\mathrm{ZnSe}$ related peaks due to the low $\mathrm{Mn}^{2+}$ dopant concentration present. ${ }^{[18]}$ However, it was observed that the presence of these surface defects during the synthesis of the Mn-ZnSe doped QDs appears to favor the generation of white light after the incorporation of the $\mathrm{Cu}$ ions into the host lattice in the later step. The emission intensity at $585 \mathrm{~nm}$ is observed to first increase gradually during annealing and then gradually decreases. This indicates the diffusion of the $\mathrm{Mn}$ ions from the center towards the surface of the nanoparticles (see Supporting Information Figure S1). In the second step, the temperature is lowered to $180^{\circ} \mathrm{C}$ so as to arrest the growth of the Mn-ZnSe doped QDs and to this the required amount of $\mathrm{Cu}$ precursor is injected. Subsequently, due to the gradual increase of the temperature, $\mathrm{Cu}$ ions were adsorbed onto the surface of the previously grown $\mathrm{Mn}-\mathrm{ZnSe}$ doped QDs, which act as small hosts for the adsorption of the $\mathrm{Cu}$ ions.

The adsorption of the $\mathrm{Cu}$ ions onto the surface of the Mn$\mathrm{ZnSe}$ nanocrystals is confirmed by the appearance of the highly intense blue-green peak at $485 \mathrm{~nm}$ (Figure $1 \mathrm{a}$ ), which is $15 \mathrm{~nm}$ red-shifted with respect to the pure $\mathrm{ZnSe}$ QD defect related emission. This peak is assigned to the recombination of an excited electron in the conduction band/defect states of the $\mathrm{ZnSe}$ host nanocrystal with the d-orbital hole of a $\mathrm{Cu}$ ion. ${ }^{[14,20,21]}$ A decrease in the band-edge emission peak intensity of the host $\mathrm{ZnSe}$ is also observed, which is a further indication for the adsorption of the $\mathrm{Cu}$ ions onto the surface of the host Mn-ZnSe nanocrystals, where the loosely adsorbed dopant ions can easily act as surface traps to quench the host PL. Interestingly, there is an accompanying decrease in the PL intensity of the $585 \mathrm{~nm}$ peak observed. The partial quenching of the highly intense $\mathrm{Mn}^{2+}$ dopant emission and appearance of the broad emission at $490 \mathrm{~nm}$ after the introduction of $\mathrm{Cu}$ ions indicates the successful adsorption of these ions onto the Mn-ZnSe doped QDs surface, which displays the opening of the more favorable optical relaxation path through the $\mathrm{Cu}$ states for the photophysical processes.

There are a number of different transition mechanisms involved in the emission of the co-doped $\mathrm{ZnSe}$ system. In Mn$\mathrm{ZnSe}$, after electrons have been excited from the valence band to the conduction band, a number of electrons may relax to the defect states, from where they can recombine with holes in the valence band, with the resultant emission of photons in the $470 \mathrm{~nm}$ spectral region. When the copper ions are present, they are adsorbed on the surface of the doped QDs and hence populate these surface sites. Therefore radiative recombination between electrons in the defect states and holes in the dorbitals of the copper ions is expected to increase in dominance. Hence significantly, the peak intensity of the copper-related PL increased by manyfold compared with both the defect- and $\mathrm{Mn}^{2+}$-related $\mathrm{d}-\mathrm{d}$ emissions. Finally, when the $\mathrm{ZnSe}$ shell is synthesized on the surface of the doped QDs, it can act to reduce the concen-
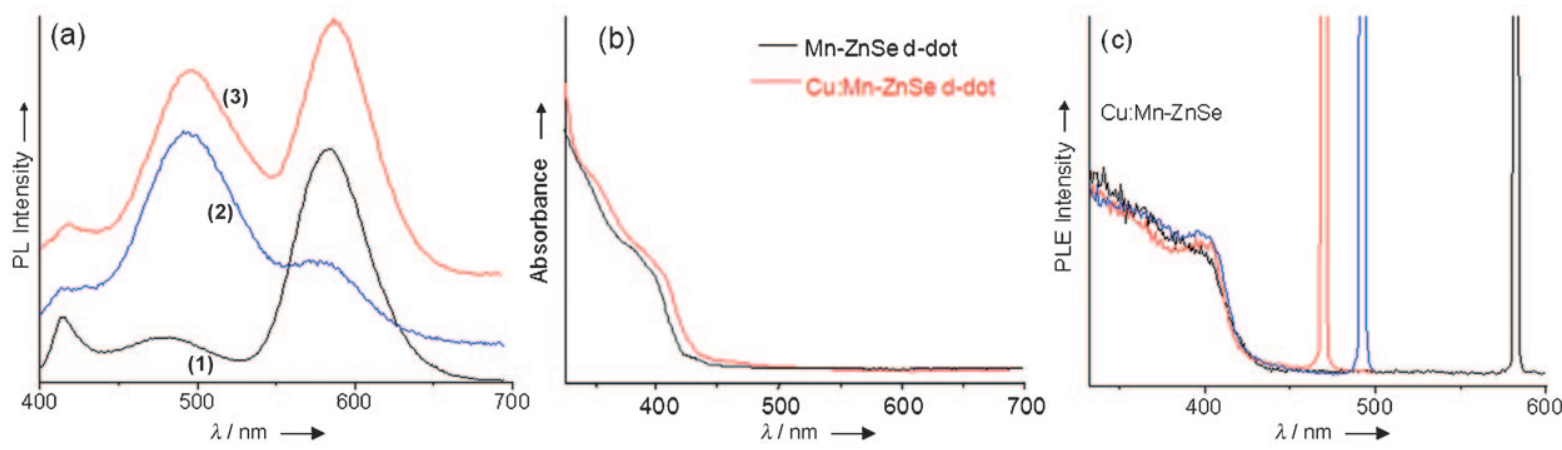

Figure 1. a) PL spectra of the samples at different steps of synthesis: 1) Mn-ZnSe doped QDs, 2) Mn-ZnSe doped QDs after Cu injection, and 3) Cu:Mn-ZnSe white-light emitting doped QDs. b) UV/Vis absorbance spectra of Mn-ZnSe and Cu:Mn-ZnSe doped QDs. c) Photoluminescence excitation spectra of the $\mathrm{Cu}: \mathrm{Mn}-\mathrm{ZnSe}$ sample taken at the different peak emission positions. 
tration of surface states and hence favor the energy transfer from the $\mathrm{ZnSe}$ host to the Mn centers.

Successful formation of the $\mathrm{ZnSe}$ shell is confirmed by the $\mathrm{Cu}$ related PL emission being red shifted (Figure $1 \mathrm{a}$ ) after the shell formation due to the accompanying increase in the size of the doped QDs. From the optical absorbance spectra (Figure $1 \mathrm{~b}$ ) a red shift in the spectrum for the $\mathrm{Cu}: \mathrm{Mn}-\mathrm{ZnSe}$ sample is observed which is additional support for the formation of the $\mathrm{ZnSe}$ shell. Photoluminescence excitation (PLE) spectra (Figure 1c) for the WLE-doped QDs taken at the different emission peak positions were found to mirror the $\mathrm{UV} / \mathrm{Vis}$ absorption spectrum of the $\mathrm{ZnSe}$ host matrix remarkably well, indicating that all emissions originated from the same particle set and not from extraneous species or undesired secondary growth of quantum dots formed during the synthesis.

From the TEM observation (Figure 2a,b) the size of the $\mathrm{Mn}-\mathrm{ZnSe}$ doped QDs and the $\mathrm{Cu}: \mathrm{Mn}-\mathrm{ZnSe}$ doped QDs were measured to be $4.0 \mathrm{~nm} \pm 10 \%$ and $5.2 \mathrm{~nm} \pm 10 \%$, respec-

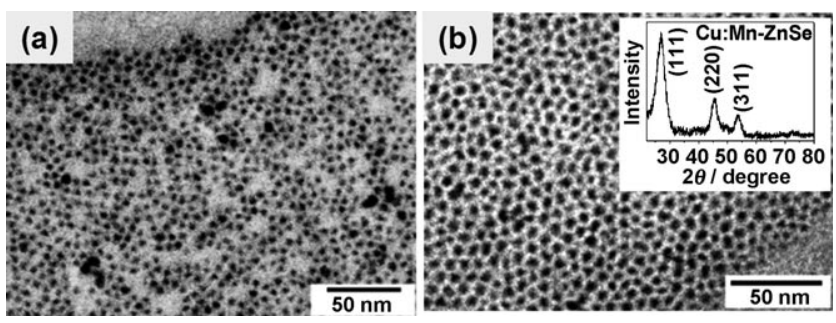

Figure 2. TEM images of a) Mn-ZnSe doped QDs, b) Cu:Mn-ZnSe doped QDs. Inset: XRD spectrum of Cu:Mn-ZnSe doped QDs.

tively, which is also in good agreement with the sizes as calculated from the absorbance spectra. A HRTEM image of the $\mathrm{Cu}: \mathrm{Mn}-\mathrm{ZnSe}$ doped QDs is provided in the Supporting Information (Figure S2). The X-ray diffraction (XRD) pattern of a typical $\mathrm{Cu}: \mathrm{Mn}-\mathrm{ZnSe}$ doped QD sample (inset of Figure $2 \mathrm{~b}$ ) displays the peak broadening, which is characteristic of a nanocrystalline material and exhibits a number of well-resolved peaks that can be indexed to the (111), (220), and (311) planes of zinc blende ZnSe (JCPDS 37-1463) and the diffractogram is devoid of any signatures which may be assigned to other phases. The size of the nanocrystals was calculated using Debye-Scherrer formula using the (111) reflection of the XRD pattern and the average particle size estimated was $5.5 \mathrm{~nm}$, which is also in good agreement with the optical study.

To further establish the inclusion of both the dopant ions in a single nanoparticle, a number of contrastive experiments were undertaken. When separately prepared $\mathrm{Mn}-\mathrm{ZnSe}$ and $\mathrm{Cu}-\mathrm{ZnSe}$ doped QDs were mixed together, no quenching of the $\mathrm{Mn}$ related emission at $585 \mathrm{~nm}$ is observed (see Figure S3). When this mixed $\mathrm{Mn}-\mathrm{ZnSe} / \mathrm{Cu}-\mathrm{ZnSe}$ doped QD solution was annealed at $260^{\circ} \mathrm{C}$ for few minutes, no drastic quenching in the Mn-ion related PL peak is observed. In contrast, after $\mathrm{Cu}$ injection during a normal synthesis, it was observed that the Mn-related emission is almost completely suppressed after $10 \mathrm{~min}$ when the temperature is increased gradually to $240^{\circ} \mathrm{C}$ (see Figure S4). This may be due to the migration of the Mn ions towards the surface of the doped QDs at this higher temperature, giving rise to a photophysical interaction between the two different ions due to the increase in their proximity. From this observation it may be postulated that the different ions are required to be separated by a minimum distance if they are to emit their individual colors. When they are close enough the $\mathrm{Cu}$ emission is observed to be dominant and the Mn-related emission is almost suppressed. Further study on this aspect will provide a better understanding of the interaction between two emitting ions occupying the same nanosized host matrix with respect to their relative positions. This also provides additional weight to previous reports that the two different dopants are not homogeneously distributed throughout the doped QDs but rather that the core may be $\mathrm{Mn}$-rich ${ }^{[22]}$ and the surface rich in $\mathrm{Cu}$ ions, and migration of the ions to within close proximity of each other is to be avoided if one is to achieve WLE.

The quantum yield (QY) of the samples at different stages of the synthesis was measured. In the first step, the QY of the $\mathrm{Mn}-\mathrm{ZnSe}$ is observed to be $22 \%$. When the $\mathrm{Cu}$ ions were adsorbed on the surface of the Mn-ZnSe doped QDs the QY is decreased to $8-10 \%$. This may be due to the generation of the surface defect states and so also the observed quenching of the highly intense $\mathrm{Mn}^{2+}$-related emission. As the emission from $\mathrm{d}-\mathrm{d}$ transitions of $\mathrm{Mn}^{2+}$ are largely affected by the crystal field and sensitive to the environment, ${ }^{[2,24]}$ these studies also give a clear indication for the interaction of the optical active channels due to the different dopant ions inside a single crystal. After adding the $\mathrm{ZnSe}$ shell in the third step, the QY was observed to increase to $13-17 \%$ under suitable conditions. Hence, in this rather complex photophysical system, the inorganic capping leads to a reduced escape possibility for the charge carriers with a consequent increase in the emission QY.

The doped QDs so obtained were also observed to be soluble in a number of organic solvents such as toluene and hexane. A white powder could be obtained by precipitating the doped QDs with acetone and drying under a nitrogen flow and was found to be stable under normal atmospheric conditions for days or for months under inert atmosphere. To further explore the photostability of the doped QDs, a solution in toluene was exposed to irradiation from the $365 \mathrm{~nm}$ line (intensity approximately $1 \mathrm{~mW} \mathrm{~cm}^{-2}$ ) of a UV lamp. Upon exposure to UV-light, the PL intensity of the dopant emission peaks decreased gradually and the $\mathrm{ZnSe}$ emission band increased. After 3 h of exposure the $\mathrm{Cu}$-related emission peak is observed to be diminished (Figure S6) which may be due to the photooxidation of $\mathrm{Cu}$ ions close to the surface of the doped QDs and also to possible gradual lattice ejection of some of the dopants from the host material under irradiation. ${ }^{[25]}$ When the doped QDs were transferred to a $5 \%$ PMMA solution in toluene and spin-coated, the resulting thin films were found to be stable (inset of Figure S5) suggesting that for applications, encapsulation methods can provide a greater degree of stability. In Figure $3 \mathrm{a}$ the white-light emission from the powders under the $365 \mathrm{~nm}$ excitation line of a UV lamp can be seen. 

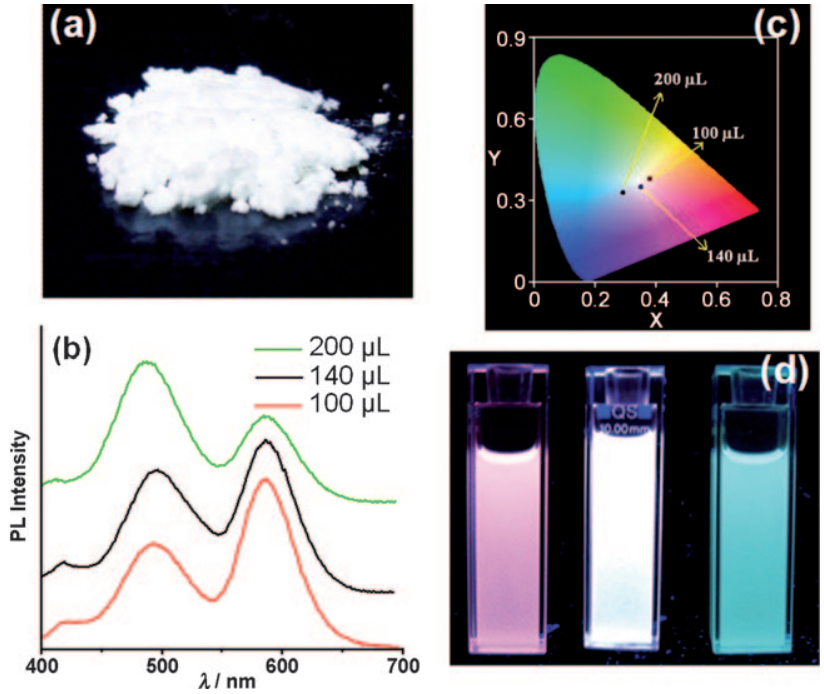

Figure 3. a) Cu:Mn-ZnSe doped QD powder showing white-light emission under a UV lamp with a $365 \mathrm{~nm}$ excitation. b-d) Cu:Mn-ZnSe doped QDs samples with different amount of Cu precursors: b) emission spectra, c) CIE coordinates, and d) photographs under a UV lamp with a $365 \mathrm{~nm}$ excitation.

A number of large-scale syntheses have also been successfully carried out and gram quantities of high-quality WLE doped QDs could be supplied from a single batch. The spectral properties of the white light could also be controllably adjusted, by systematically varying the ratio of the dopant concentrations. Figure $3 \mathrm{~b}$ presents the emission profiles recorded for the samples obtained after different amounts of the $\mathrm{Cu}$ precursor were injected. Figure $3 \mathrm{c}$ shows the corresponding CIE coordinates and Figure $3 \mathrm{~d}$ photographs of the emission under UV light for the same samples. For these samples the CIE coordinates, color rendering index, and correlated color temperature have been included in tabular form in the Supporting Information. This therefore demonstrates the added possibility to tune the photometric properties of WLE for different specific commercial applications as may be so required. In Table S3 the shades of WLE are shown to be conveniently spanned using varying amounts of the $\mathrm{Cu}$ precursor. When the emission colors from the doped QDs are compared with the different color temperatures (warm vs. cold white) of commercial white-light LEDs, the emission from the doped QDs presented here belongs to the cold white-light classification.

Time-resolved spectroscopy measurements were undertaken in order to gain insight into the various mechanisms involved in the emission process. Figure 4 shows the photoluminescence decay profiles of the different emission centers in the $\mathrm{Mn}-\mathrm{ZnSe}$ and $\mathrm{Cu}: \mathrm{Mn}-\mathrm{ZnSe}$ materials. The lifetimes associated with the various decay processes have also been presented in tabular form in the Supporting Information. By the introduction of the $\mathrm{Cu}$ d-states, it is observed that the band-edge emission is quenched and thus a faster decay results, while the trap-associated recombination becomes slower as is apparent at the tail end of the decay profiles. This is attributed to the enhanced efficiency of the trap-state population in the presence of the $\mathrm{Cu}$ ions. For both samples the exciton emission and trap emission are fast processes compared to the emission from the doped sites. The emission collected from $\mathrm{Cu}: \mathrm{Mn}-\mathrm{ZnSe}$ at $470 \mathrm{~nm}$ most probably contains a contribution which may be associated with trap emission, as indicated by the shorter component in the lifetime fitting parameters observed for the Mn-ZnSe doped QDs (see Supporting Information, Tables S1 and S2). However, the emission recorded at $495 \mathrm{~nm}$ displayed a dominant decay channel of ca. $100 \mathrm{~ns}$ in the lifetime profile, which may be attributed to the $\mathrm{Cu}$ d-states, as it is unlikely to be due to either excitonic or purely trap emission, both of which are intrinsically much faster processes.

The decay profile of the Mn-related emission at $585 \mathrm{~nm}$ is presented in Figure $4 \mathrm{c}$, and shows the mono-exponential nature of both the samples. The lifetime measured for the Mn$\mathrm{ZnSe}$ sample is calculated to be $264 \mu$ s and that for the $\mathrm{Cu}: \mathrm{Mn}-\mathrm{ZnSe}$ sample is $324 \mu$ s. The $\mathrm{Mn}^{2+}$ ion emission in the $\mathrm{Cu}: \mathrm{Mn}-\mathrm{ZnSe}$ doped QDs displayed a longer lifetime due to the thicker ZnSe overcoating layer, which also supports the successful formation of a thicker shell of $\mathrm{ZnSe}$ around the doped QDs encapsulating both the $\mathrm{Mn}$ and $\mathrm{Cu}$ ions. Based on these results, the energy level diagram for the doped QDs may be depicted as shown in Figure 5. This system of multiple dopants, which are very close to each other and have lifetime values differing by three orders of magnitude, also raises certain questions concerning the relative emission efficiency between two emission bands and future studies providing such insights will be helpful in explaining the complex nature of the energy states.
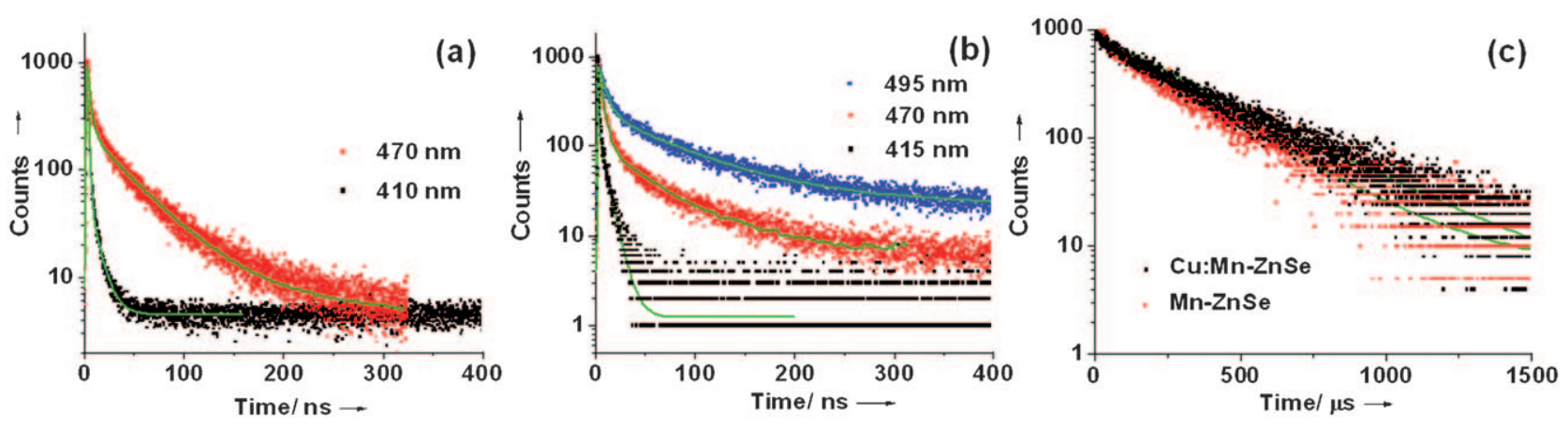

Figure 4. Photoluminescence (PL) decay traces of a) Mn-ZnSe doped QDs, b) Cu:Mn-ZnSe doped QDs at different peak positions, and c) of $\mathrm{Cu}: \mathrm{Mn}-\mathrm{ZnSe}$ and $\mathrm{Mn}-\mathrm{ZnSe}$ doped QDs at $585 \mathrm{~nm}$. 


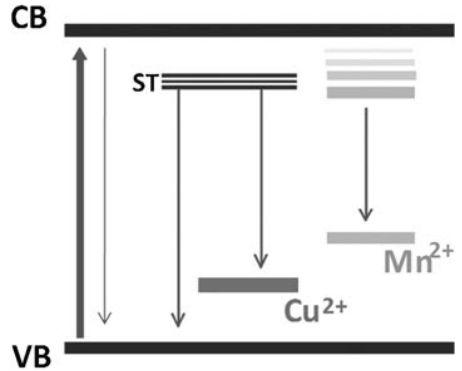

Figure 5. Energy level diagram for the Cu:Mn-ZnSe doped QDs. (VB: valence band, $\mathrm{CB}$ : conduction band, ST: surface trap states).

In conclusion, we report the generation of doubly doped QDs by a hot-injection colloidal synthesis approach that possess high-quality white-light emission in both the colloidal solution and solid-state powder with photoluminescence efficiencies as high as $17 \%$. This protocol has proven to be robust and offers the possibility of being extended to other host materials allowing co-doping with different dopants to obtain high quality emitters that have the potential to cover most of the visible and NIR spectral window for future optoelectronic applications. The WLE-doped QDs may facilitate simple device implementation and hold great promise for the future of solid-state lighting. In addition, these doped QDs may offer high-performance emissive materials without the inclusion of any of the highly toxic class-A elements $(\mathrm{Cd}, \mathrm{Hg}$, and $\mathrm{Pb})$ and therefore may be used in place of the current workhorse of intrinsic quantum dot emitters which are based on these materials.

Received: January 19, 2011

Published online: April 7, 2011

Keywords: luminescence $\cdot$ nanomaterials - photophysics . quantum dots $\cdot$ white light emission

[1] M. J. Bowers II, J. R. McBride, S. J. Rosenthal, J. Am. Chem. Soc. 2005, 127, 15378.

[2] S. Sapra, S. Mayilo, T. A. Klar, A. L. Rogach, J. Feldmann, $A d v$. Mater. 2007, 19, 569

[3] C. C. Shen, W. L. Tseng, Inorg. Chem. 2009, 48, 8689.

[4] A. A. Bol, A. Meijerink, Phys. Chem. Chem. Phys. 2001, 3, 2105.

[5] K. W. Cheah, L. Ling, X. Huang, Nanotechnology 2002, 13, 238.
[6] H. S. Chen, S. J. J. Wang, C. J. Lo, J. Y. Chi, Appl. Phys. Lett. 2005, 86, 131905.

[7] a) S. Nizamoglu, E. Mutlugun, T. Ozel, H. V. Demir, S. Sapra, N. Gaponik, A. Eychmüller, Appl. Phys. Lett. 2008, 92, 113110; b) H. V. Demir, S. Nizamoglu, E. Mutlugun, T. Ozel, S. Sapra, N. Gaponik, A. Eychmüller, Nanotechnology 2008, 19, 335203; c) A. Rizzo, Y. Li, S. Kudera, F. D. Sala, M. Zanella, W. J. Parak, R. Cingolani, L. Manna, G. Gigli, Appl. Phys. Lett. 2007, 90, 051106.

[8] a) Q. Dai, C. E. Duty, M. Z. Hu, Small 2010, 6, 1577; b) H. Sun, J. Zhang, H. Zhang, Y. Xuan, C. Wang, M. Li, Y. Tian, Y. Ning, D. Ma, B. Yang, Z. Y. Wang, ChemPhysChem 2006, 7, 2492; c) X. Liu, Y. Jiang, C. Wang, S. Li, X. Lan, Y. Chen, Phys. Status Solidi A 2010, 207, 2472; d) H. S. Jang, H. Yang, S. W. Kim, J. Y. Han, S. G. Lee, D. Y. Jeon, Adv. Mater. 2008, 20, 2696; e) Y. Li, A. Rizzo, R. Cingolani, G. Gigli, Adv. Mater. 2006, 18, 2545.

[9] V. Wood, J. E. Halpert, M. J. Panzer, M. G. Bawendi, V. Bulovic, Nano Lett. 2009, 9, 2367.

[10] N. Pradhan, D. M. Battaglia, Y. Liu, X. Peng, Nano Lett. 2007, 7, 312.

[11] N. C. Greenham, X. Peng, A. P. Alivisatos, Phys. Rev. B 1996, 54, 17628.

[12] P. V. Radovanovic, D. R. Gamelin, Phys. Rev. Lett. 2003, 91, 157202.

[13] S. C. Erwin, L. Zu, M. I. Haftel, A. L. Efros, T. A. Kennedy, D. J. Norris, Nature 2005, 436, 91 .

[14] N. Pradhan, D. Goorskey, J. Thessing, X. Peng, J. Am. Chem. Soc. 2005, 127, 17586

[15] a) D. J. Norris, N. Yao, F. T. Charnock, T. A. Kennedy, Nano Lett. 2001, 1, 3; b) C. Graf, A. Hofmann, T. Ackermann, C. Boeglin, R. Viswanatha, X. Peng, A. F. Rodríguez, F. Nolting, E. Rühl, Adv. Funct. Mater. 2009, 19, 2501.

[16] a) J. Xue, Y. Ye, F. Medina, L. Martinez, S. A. Lopez-Rivera, W. Giriat, J. Lumin. 1998, 78, 173; b) S. E. Irvine, T. Staudt, E. Rittweger, J. Engelhardt, S. W. Hell, Angew. Chem. 2008, 120, 2725; Angew. Chem. Int. Ed. 2008, 47, 2685.

[17] A. Nag, D. D. Sarma, J. Phys. Chem. C 2007, 111, 13641.

[18] Z. Quan, D. Yang, C. Li, D. Kong, P. Yang, Z. Cheng, J. Lin, Langmuir 2009, 25, 10259.

[19] X. Lü, J. Yang, Y. Fu, Q. Liu, B. Qi, C. Lu, Z. Su, Nanotechnology 2010, 21, 115702

[20] J. F. Suyver, T. V. Beek, S. F. Wuister, J. J. Kelly, and A. Meijerink, Appl. Phys. Lett. 2001, 79, 4222.

[21] G. B. Stringfellow, R. H. Bube, Phys. Rev. 1968, 171, 903.

[22] N. Pradhan, X. Peng, J. Am. Chem. Soc. 2007, 129, 3339.

[23] R. N. Bhargava, D. Gallagher, X. Hong, A. Nurmikko, Phys. Rev. Lett. 1994, 72, 416.

[24] S. Wang, S. Westcott, W. Chen, J. Phys. Chem. B 2002, 106, 11203.

[25] D. Chen, R. Viswanatha, G. L. Ong, R. Xie, M. Balasubramaninan, X. Peng, J. Am. Chem. Soc. 2009, 131, 9333. 